\title{
Modeling and Algorithmic Challenges in Online Social Networks
}

\author{
Ravi Kumar \\ Yahoo! Research, 701 First Ave., Sunnyvale, CA 94089, USA \\ ravikumar@yahoo-inc.com
}

\begin{abstract}
Online social networks have become major and driving phenomena on the web. In this talk we will address key modeling and algorithmic questions related to large online social networks. From the modeling perspective, we raise the question of whether there is a generative model for network evolution. The availability of time-stamped data makes it possible to study this question at an extremely fine granularity. We exhibit a simple, natural model that leads to synthetic networks with properties similar to the online ones. From an algorithmic viewpoint, we focus on challenges posed by the magnitude of data in these networks. In particular, we examine topics related to influence and correlation in user activities and compressibility of such networks.
\end{abstract}

